\title{
THE NUMBER DENSITY OF QUASARS AS A PROBE OF INITIAL POWER SPECTRUM ON SMALL SCALE
}

\author{
B. Novosyadlyj, Yu. Chornij \\ Astronomical Observatory of Lviv State University, 8 Kyrylo i Methodij Str., Lviv, UA-290005, Ukraine
}

(Received November 22, 1996)

\begin{abstract}
The dependence of the number density of the bright QSOs at different redshifts $\left(n_{Q S O}(z)\right)$ on the initial power spectrum is studied. It is assumed that the QSO phenomenon is an early short term stage of evolution of massive galaxies with $M \geq 2 \times 10^{11} h^{-1} M_{\odot}$. The duration of such a QSO stage which is passed through by fraction $\alpha$ of galaxies is determined by means of minimization of the divergence of the theoretical number density of QSOs at different redshifts for specified initial spectrum from the observable one [1]. It is shown that the nearest number densities of QSOs at $0.7 \leq z \leq 3.5$ to observable ones are obtained for the tilted CDM model $\left(\Omega_{b}=0.1, n=0.7\right)$. The QSO stage lasts $\sim 7 \times 10^{7} / \alpha$ years and begins soon after the moment of the rise of the first counterflow in collisionless component and of the shock wave in gas.

The possibility of the reconstruction of the initial power spectrum on a small scale on the basis of the observable data on the number density of QSOs at different $z$ is considered too. Such reconstructed spectrum in comparison with the standard CDM has steep reducing of power at $k>0.5 h M p c^{-1}$.

Key words: cosmology, initial power spectrum, dark matter, quasars.
\end{abstract}

PACS number(s): 98.80.Ft, 98.54.Aj, 98.65.Dx

\section{INTRODUCTION}

The problem of the formation of galaxies and large scale structure of the Universe has an astrophysical aspect consisting in the search for a connection between the properties of luminous objects and dark matter halo, as well as a cosmological aspect consisting in the determination of the initial power spectrum of density fluctuations. Obtaining such spectrum from first principals would essentially simplify the solving of the problem on the whole. Unfortunately, today we can not surely say what the postinflation spectrum is like the scale invariant of $P_{p i}=A k^{n}$ with $n=1$, tilted with $n \neq 1$, or more complicated. The nature of dark matter (baryonic, collisionless cold dark matter, futher CDM, hot dark matter, futher HDM, or their mixture $\mathrm{H}+\mathrm{CDM}$, or other), and values of the cosmological parameters $h \equiv \frac{H_{0}}{100 \mathrm{~km} / \mathrm{s} \mathrm{Mpc}}$ ( $H_{0}$ is the Hubble constant), $\Omega_{t} \equiv \frac{8 \pi G}{3 H_{0}^{2}} \rho_{t}$ ( $\rho_{t}$ is total mean density), $\Omega_{b} \equiv \frac{8 \pi G}{3 H_{0}^{2}} \rho_{b}$ ( $\rho_{b}$ is mean density of baryons) and the cosmological constant $\Lambda$ remain uncertain. Spectra more preferable from the theoretical point of view (standard CDM model, tilted $\mathrm{CDM}, \mathrm{CDM}+\Lambda$ or hybrid $\mathrm{H}+\mathrm{CDM}$ ) give predictions consistent with the observable data marginally only [2]- [9]. Furthermore, the different spectra normalised to the COBE r.m.s. cosmic microwave background (CMB) anisotropy predict close characteristics of the large scale structure of the Universe, e.g. $\Delta T / T$ at degree angular scale, large scale peculiar velocity field, etc. $[6,8,9]$ so that it is impossible to distinguish them. Therefore, it is very important to build up the new cosmological tests based on the extension of a class of objects with predicted observational manifestations sensitive to the initial power spectrum on a small scale. Here we shall investigate the dependence of the number density of quasars (QSOs) at different redshifts against the initial power spectra. To make it possible we suppose that a QSO is the early short term stage of evolution of a massive galaxy and appears in the corresponding peaks of initial random Gaussian field of density fluctuations. The number density of the already collapsed peaks of a certain scale at different redshifts $z$ strongly depends on an amplitude and slope of the power spectrum of such a field at small scales $\left(k \sim 0.1-10 h M p c^{-1}\right)$.

Such an approach was applied independently by some authors. For example, Cen et al. [5] have calculated the comoving number density of nonlinear objects as a function of the redshift $\mathrm{z}$ using Press-Schechter approximation [10]. Their results show that an observable number density of quasar-like objects between $2 \leq z \leq 4$ can be explained by tilted CDM with $n \geq 0.7$ and a mass of host galaxies $\leq 510^{12} M_{\odot}$. Blanchard et al. [11] tested HDM models and have shown the disability of these models to explain the observable concentration of QSOs at $z \sim 3-4$ when primordial scale-invariant spectrum $(n=1)$ is normalised to the COBE r.m.s. cosmic microwave background anisotropy [12]. Kashlisky [13] tested the hybrid $\mathrm{H}+\mathrm{CDM}$ and modified CDM models by the number density of distant QSOs at $z=4.5$. The number density of the peaks-precursors of massive galaxies $\left(M>10^{11} h^{-1} M_{\odot}\right)$, which passed a quasars stage soon after their central regions had collapsed, was calculated for the different spectra. The mean number density of QSOs predicted by the modified CDM model with $\Omega h=0.1,0.2,0.3$ and $\mathrm{H}+\mathrm{CDM}$ was found to be less than the observable one $n_{Q S O}^{o b s} \approx 400 h^{3} G p c^{-3}$ at $z=4.5$. Nusser \& Silk [14] for finding $n_{Q S O}(z)$ in redshift range 
2-5 for a given spectrum used the Gaussian peaks formalism by Bardeen et al. [15] and showed that this formalism is more preferable than the Press-Schechter one. They assumed the number density of very bright QSOs $\left(L \geq 10^{47} \mathrm{erg} / \mathrm{s}\right)$ to be equal to the number density of peaks with the masses $M \geq 2 \times 10^{11} h^{-1} M_{\odot}$ within a certain height interval corresponding to the physically substantiated duration of quasar stage $\left(\approx 4 \times 10^{7}\right.$ years $)$. They have concluded that the tilted CDM model with $n=0.8, \mathrm{H}+\mathrm{CDM}\left(\Omega_{C D M} \approx 0.7, \Omega_{H D M} \approx 0.3\right)$ and the scaled $\mathrm{CDM}$ with $0.2 \leq \Omega h \leq 0.3$ are compatible with the mean abundance of quasars at $2 \leq z \leq 5$. Ma \& Bertchinger [16] have carried out the numerical simulation of the formation of elements of a large scale structure of the Universe with masses $10^{11}-10^{13} M_{\odot}$ in hybrid $\mathrm{H}+\mathrm{CDM}$ models and analysed the possibility of explaining the observable number density of QSOs at $2.2 \leq z \leq 4.5$ in the framework of the Press-Schechter formalism. They have shown that the H+CDM model with the $\Omega_{H D M}=0.2$ predicts $n_{Q S O}(z)$ which matches the observable one if quasars are created soon after the collapse of nearly spherical peaks.

The aim of the paper is an improvement of this approach to the explanation of the observable data on the abundance of the bright QSOs in a wider range of $z=0.7 \div 4.7[1,17]$, testing the preferable initial spectra, which marginally match other observable data on a large scale structure of the Universe. It searches the spectrum momenta to reproduce the observable data on $n_{Q S O}(z)$ constructing the suitable phenomenological spectrum on a small scale.

\section{THE MAIN ASSUMPTIONS AND METHOD}

We explore the distribution of the quasar abundance over redshift $n_{Q S O}(z)$ within the framework of the theory of the formation of a large scale structure of the Universe in the peaks of random Gaussian field of scalar density fluctuations as a consequence of the gravitational instability on the Einstein-de Sitter cosmological background. In this approach galaxies and objects of other scales are formed in the peaks of density fluctuations which have an amplitude of

$$
\delta \equiv \frac{\delta \rho}{\rho} \geq \nu_{t h} \sigma_{0}\left(R_{f}\right)
$$

where $\sigma_{0}$ is r.m.s. amplitude of the fluctuations of a certain scale $R_{f}, \nu_{t h}$ is some threshold height which corresponds to the minimal amplitude when objects of this scale are forms still.

In the theory of random Gaussian fields [15,18] a comoving number density of the peaks with the height of $\nu \geq \nu_{t h}$ is

$$
n\left(\nu_{t h}\right)=\int_{0}^{\infty} p\left(\nu ; \nu_{t h}, q\right) N_{p k}(\nu) d \nu
$$

where

$$
p\left(\nu ; \nu_{t h}, q\right)=\frac{\left(\nu / \nu_{t h}\right)^{q}}{1+\left(\nu / \nu_{t h}\right)^{q}}
$$

is the threshold function, $N_{p k}(\nu)$ is the differential number density of peaks in the amplitude range of $(\nu, \nu+$ $d \nu)$, which depends on $\nu$ and the spectrum momenta $\sigma_{0}, \sigma_{1}, \sigma_{2}$ (formulae $4.3-4.5$ and A15 in the paper by Bardeen et al. [15]).

We suppose that a certain fraction of massive galaxies $\alpha_{1}$ passes through quasar stage of evolution. Maybe, only some fraction of them $\alpha_{2}$ is visible by an Earth observer. Therefore, the fraction of galaxies passing through quasars stage and visible by an Earth observer as QSOs is $\alpha \equiv \alpha_{1} \alpha_{2} \leq 1$. If we adopt the black hole model of quasars, then the minimum mass associated with a host galaxy is $\sim 10^{11}-10^{12} M_{\odot}[14,19,20]$. For the selection of galactic scale peaks with the mass of $M \geq 2 \times 10^{11} h^{-1} M_{\odot}$ we smoothed the initial random Gaussian density field $\delta(\mathbf{x})$ by the Gaussian filter function

$$
\bar{\delta}\left(\mathbf{x} ; R_{f}\right)=\frac{1}{\left(2 \pi R_{f}^{2}\right)^{1.5}} \int \delta\left(\mathbf{x}^{\prime}\right) e^{-\frac{\left(\mathbf{x}^{\prime}-\mathbf{x}\right)^{2}}{2 R_{f}^{2}}} d^{3} \mathbf{x}^{\prime}
$$

with $R_{f}=0.35 h^{-1} \mathrm{Mpc}$. It means that the power spectrum of the smoothed field is

$$
P\left(k ; R_{f}\right)=P(k) e^{-k^{2} R_{f}^{2}},
$$

where

$$
P(k) \equiv<\delta_{k} \delta_{k}^{*}>
$$

is the initial power spectrum, $\delta_{k}$ is the Fourier transformation of the $\delta(\mathbf{x})$ :

$$
\delta(\mathbf{x})=\frac{1}{(2 \pi)^{1.5}} \int \delta_{k} e^{i \mathbf{k x}} d^{3} \mathbf{k}
$$

Those among them which have an amplitude

$$
\bar{\delta}\left(\mathbf{x}, R_{f}\right) \geq \nu_{t h}^{g} \sigma_{0}
$$

where

$$
\sigma_{0}^{2}=\frac{1}{(2 \pi)^{2}} \int d k k^{2} P(k) e^{-k^{2} R_{f}^{2}}
$$

is r.m.s. amplitude of density fluctuations, evolve to massive galaxies. Their concentration is known from $C_{f} A$ catalogue [21] $n_{g}^{o b s} \approx\left(4.6 h^{-1} M p c\right)^{-3}$. Using eq. (1) for massive galaxies

$$
n\left(\nu_{t h}^{g}, q^{g}\right)=n_{g}^{o b s}
$$

one parameter of the threshold function (e.g. $\nu_{t h}^{g}$ ) can be found. 
As follows from Tollman model, a homogeneous spherical-symmetrical adiabatic fluctuation on the Einstein-de Sitter cosmological background collapses at

$$
z_{c} \equiv\left(\frac{t_{0}}{t_{c}}\right)^{\frac{2}{3}}-1=0.59 \sigma_{0} \nu_{c}-1
$$

where $t_{0} \simeq 1.3 \times 10^{10}$ years is present cosmological time (here and further we assume $H_{0}=50 \frac{\mathrm{km}}{\mathrm{sMpc}}$ ), $t_{c}$ is the moment of collapse, $\nu_{c}$ is the corresponding height of the peak. At this moment $t_{c}$ the appearance of the first counterflows in dark matter and the generation of shockwave in baryon component take place. A massive black hole in the central region of such a peak may be formed during $\sim 10^{8}$ years as it follows from the physical models of quasar mechanism (see, for example, [20]).Therefore, we suppose that QSO stage of galaxy evolution begins soon after the collapse of the central region of peak $t_{c}$ or later by a certain time interval $\Delta t$ called a time delay. Duration of the QSO stage or quasar lifetime is $\tau_{Q S O}$.

In such an approach the number density of the QSOs at the redshift $z$ is the number density of the corresponding peaks of density fluctuations (precursors of massive galaxies) which had collapsed between $t_{c}-\tau_{Q S O}$ and $t_{c}$. These peaks we can see as QSOs at the redshift $z$ corresponding to the time $t(z)=t_{c}\left(z_{c}\right)+\Delta t$ :

$$
z=\left(\frac{t_{0}}{t_{c}\left(z_{c}\right)+\Delta t}\right)^{\frac{2}{3}}-1
$$

The height of such peaks $\nu$ is in the range of $\left(\nu_{c}, \nu_{c}+\right.$ $\Delta \nu)$, where

$$
\begin{gathered}
\nu_{c}=1.69 \sigma_{0}^{-1}\left(z_{c}+1\right), \\
\Delta \nu=1.13\left(z_{c}+1\right)^{2.5} \frac{\tau_{Q S O}}{\sigma_{0} t_{0}},
\end{gathered}
$$

which follows from eq. (3). Then the number density of QSOs is

$$
n_{Q S O}(z)=\alpha \int_{\nu_{c}}^{\nu_{c}+\Delta \nu} p\left(\nu ; \nu_{t h}, q\right) N_{p k}(\nu) d \nu
$$

where $z, \nu_{c}$ and $\Delta \nu$ are defined by eq. $4,5,6$ respectively. As we can see, the number density of QSOs $n_{Q S O}(z)$ depends upon the duration of the quasar stage $\tau_{Q S O}$, the time delay $\Delta t$, parameters of the threshold function $\nu_{t h}$ and $q$, momenta of the spectrum smoothed by the galactic filter $\sigma_{0}, \sigma_{1}, \sigma_{2}$ and fraction $\alpha$ of peaks-precursors passing quasar stage and visible by Earth observer. If quasar stage is long-termed $\left(\tau_{Q S O} \sim t_{0}\right)$ then the number density of QSOs at all $z$ is equal to a fraction of the already collapsed peaks-precursors of massive galaxies visible from the Earth at quasar stage of their evolution: $n_{Q S O}(z)=\alpha n_{g}(z)$. If quasar stage is short-termed
$\left(\tau_{Q S O} \ll t_{c}\right)$, then it follows from $(7)$ and $(6)$ that

$$
n_{Q S O}(z)=1.13 \alpha \frac{\left(z_{c}+1\right)^{2.5}}{\sigma_{0} t_{0}} p\left(\nu ; \nu_{t h}, q\right) N_{p k}(\nu) \tau_{Q S O} .
$$

It is valid for the above mentioned estimation of $\tau_{Q S O} \sim$ $10^{7}-10^{8}$ years.

We suppose that the duration of the quasar stage $\tau_{Q S O}$, the time delay $\Delta t$ and the parameters of the threshold function $\nu_{t h}$ and $q$ do not depend on $z$. Also we assume that the sample of bright quasars given by Schmidt et al. [1] and Boyle et al. [17] is complete for all $z$ in the range of $0.7-4.7$. Here we do not discuss the problem concerned with the observational data but pay particular attention to the theoretical approach of their being used for cosmological problems.

Now we can calculate the number density of QSOs for a set of redshifts and given values of the duration of QSO stage $\tau_{Q S O}$, the time delay $\Delta t$, the fraction $\alpha$ of massive galaxies passing quasar stage, parameters $\nu_{t h}$ and $\mathrm{q}$ of threshold function and momenta of spectrum $\sigma_{0}, \sigma_{1}, \sigma_{2}$. For the present spectrum normalised in a certain way the momenta $\sigma_{0}, \sigma_{1}, \sigma_{2}$ are calculated independently. The time delay $\Delta t$ for a given spectrum we find in the following manner. Using eq. (8) we calculate the redshift of maximum $z_{\max }$ of the dependence $n_{Q S O}(z)$. If $z_{\max }$ is higher than the one of observable $n_{Q S O}^{o b s}(z)$ [1] $-z_{\text {max }}^{o b s} \approx 2.2$ then we find the time delay $\Delta t$ from the equation

$$
\left.\frac{d n_{Q S O}(z)}{d z}\right|_{z_{\max }^{o b s}=2.2}=0 .
$$

In those models where $z_{\max }$ is less than or equal to $z_{\text {max }}^{o b s} \approx 2.2$ we assume $\Delta t=0$. The remaining four values $\alpha, \tau_{Q S O}, \nu_{t h}$ and $q$ are unknown. Finding them by the method of minimization of divergence vector is a matter of testing the known spectra.

We try also to reconstruct the initial power spectrum from the observable data on number density of QSOs in a wide range of redshifts and massive galaxies on $z \approx 0$. For this we consider momenta of spectrum $\sigma_{0}, \sigma_{1}, \sigma_{2}$ as unknown values and find them in the same way.

In all cases we write eq. (7) for 6 points of data by Schmidt et al. [1] on the $z_{1}=4.7, z_{2}=4.05, z_{3}=3.7$, $z_{4}=3, z_{5}=2.8, z_{6}=2.2$, for 3 points on the curve by Boyle et al. [17] on $z_{7}=2.0, z_{8}=1.5, z_{9}=0.7$ and eq. (2) for the number density of massive galaxies on $z_{10}=0$ :

$$
\begin{gathered}
\alpha \int_{\nu_{i}}^{\nu_{i}+\Delta \nu_{i}} p\left(\nu ; \nu_{t h}, q\right) N_{p k}(\nu) d \nu=n_{q s o}^{o b s}\left(z_{i}\right), \\
(i=1,2 \ldots 9), \\
\int_{0}^{\infty} p\left(\nu ; \nu_{t h}, q\right) N_{p k}(\nu) d \nu=n_{g}^{o b s}
\end{gathered}
$$


where $\nu_{i}, \Delta \nu_{i}$ are calculated for $z_{i}$ according to (5) and (6) respectively. So, we solve defined above system of equations (10), (11) (or (10) only) by the method of minimization of the divergence vector using MathCad [22] software.

\section{RESULTS}

\section{A. Testing of spectra}

We shall test the preferable initial power spectra of density fluctuations on the flat Friedmanian background expected in standard CDM model $\left(\Omega_{C D M}=\right.$ $\left.0.9, \Omega_{b}=0.1\right)$, tilted CDM ones and hybrid H+CDM one $\left(\Omega_{C D M}=0.6, \Omega_{H D M}=0.3, \Omega_{b}=0.1\right)$, which marginally match other observational data on a large scale structure of the Universe [2]- [9]. The transfer function $T(k)$ in the spectra $P(k)=A k^{n} T^{2}(k)$, where $A$ is constant of normalisation [43], is taken from the paper by Holtzman [23]. We normalised all spectra to produce the COBE data on CMB temperature anisotropy at angular scales $10^{\circ}[24,25]:\left\langle\left(\frac{\Delta T}{T}\right)^{2}\right\rangle^{\frac{1}{2}}=(1.1 \pm 0.1) \times 10^{-5}$. We calculate $\left\langle\left(\frac{\Delta T}{T}\right)^{2}\right\rangle=C\left(0 ; 10^{\circ}\right)$ using the approximation formula by Wilson and Silk [26] for the correlation function of CMB temperature anisotropy measured by receiver with Gaussian response function and exact formula $[27,28]$ for the correlation function $C(\alpha) \equiv<$ $\frac{\Delta T}{T}(0) \frac{\Delta T}{T}(\alpha)>$. Both Sachs-Wolfe and Doppler effects are taken into account (see also [28]). The contribution of tensor mode to $\Delta T / T$ is different in different models of inflation. In most of them it is not dominating at the COBE angular scale (see review by [29] and references cited therein), in others it is neg- ligible even if $n<1$ (for example "natural" inflation, [30]). In this work, like the ones cited here, we consider only models of inflation which give no significant tensor mode. But one can easily cross over to models of inflation with significant tensor mode when the ratio $\alpha_{T} \equiv(\Delta T / T)_{T} /(\Delta T / T)_{S}$ is known (here $(\Delta T / T)_{T}$ and $(\Delta T / T)_{S}$ is the contribution of tensor and scalar modes correspondingly). The constant of normalization then will be $A^{\prime}=A /\left(1+\alpha_{T}^{2}\right)$, the momenta of spectrum $\sigma_{j}^{\prime}=\sigma_{j} / \sqrt{1+\alpha_{T}^{2}}$, peaks-precursors of QSOs will be collapsed at $z_{c}^{\prime}=\left(z_{c}+1\right) / \sqrt{1+\alpha_{T}^{2}}-1$, where the values without $\left({ }^{\prime}\right)$ are the ones calculated without the tensor mode. Parameters of cosmological models, constants of normalisation $A$ of the power spectra, their momenta $\sigma_{0}, \sigma_{1}, \sigma_{2}$ and biasing parameters $b \equiv 1 / \sigma_{8}$, where $\sigma_{8}$ is the r.m.s. mass fluctuations in the top-hat sphere with the radius $8 h^{-1} M p c$, are presented in table 1 . (For comparison, our normalization constant A for CDM model agrees with analogous constant from paper [31] reduced to $\mathrm{h}=0.5$ and $\sigma\left(10^{\circ}\right)=30.5 \mu K$ with the accuracy better then $0.1 \%$ ).

\begin{tabular}{|c|c|c|c|c|}
\hline & \multicolumn{3}{|c|}{ CDM } & $H+C D M$ \\
\hline$n$ & 1 & 0.8 & 0.7 & 1 \\
\hline$A$ & $7.33 \times 10^{6}$ & $1.62 \times 10^{6}$ & $7.57 \times 10^{5}$ & $7.51 \times 10^{6}$ \\
$\sigma_{0}$ & 4.78 & 2.37 & 1.67 & 1.39 \\
$\sigma_{1}$ & 4.77 & 2.18 & 1.47 & 0.91 \\
$\sigma_{2}$ & 7.93 & 3.50 & 2.31 & 1.32 \\
$b$ & 1.05 & 1.77 & 2.30 & 1.66 \\
\hline
\end{tabular}

Table 1. The constant of normalisation of the spectra, their momenta $\sigma_{0}, \sigma_{1}, \sigma_{2}$ on the galactic scale $\left(R_{f}=0.35 h^{-1} M p c\right)$ and the biasing factor $b$, defined by $b=\sigma_{8}^{-1}$, where $\sigma_{8}$ is r.m.s. mass fluctuation on the scale of $8 h^{-1} M p c$.

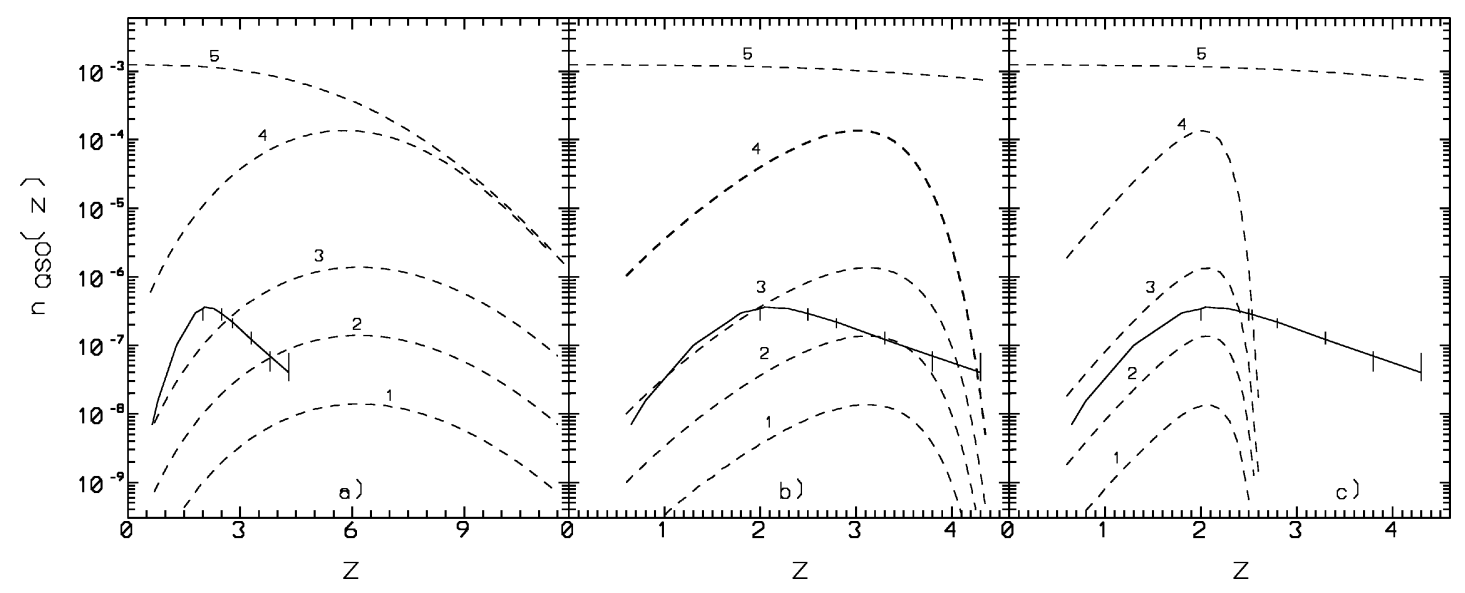

Fig. 1. Quasar abundance against redshift for different values of the $\tau_{Q S O}=10^{4}, 10^{5}, 10^{6}, 10^{8}, 10^{10}$ years (dashed lines $1,2,3,4,5$ respectively in each panel) and time delay $\Delta t=0$ (a), $\Delta t=10^{9}$ years (b), $\Delta t=1.61 \times 10^{9}$ years (c) for CDM spectrum with $n=1, \Omega_{C D M}=0.9$ and $\Omega_{b}=0.1$. The solid line with error bars is the comoving space density of quasars from Schmidt et al. (1991). The upper dashed line (5) is the number density of the already collapsed peaks-precursors of bright galaxies. In all cases $\alpha=1$. 
For beginning we analyse the dependence $n_{Q S O}(z)$ on $\tau_{Q S O}, \Delta t, \nu_{t h}$ and $q$ for different spectra accepting $\alpha$ equal to 1 . The results are presented in fig. 1-3. As we can see, the number density of QSOs is proportional to $\tau_{Q S O}$, meanwhile the time delay displaces the maximum of $n_{Q S O}$ to a lower $z$ (fig. 1). Reducing the power spectrum at small scale results in a steeper declination of $n_{Q S O}(z)$ at high $z$ and displacing its maximum to a lower $z$ (fig. 1-2).

Increasing the value of the threshold parameter $q$ $\left(\tau_{Q S O}, \nu_{t h}\right.$ are constants) reduces the number density of QSOs at low redshift and displaces its maximum to a high $z$ (fig. 3a). The dependence of $n_{Q S O}(z)$ on $\nu_{t h}$ ( $\tau_{Q S O}$ and $q$ are constants) is similar (fig. $3 \mathrm{~b}$ ). Increasing $q$ with finding the corresponding $\nu_{t h}$, which ensures $n_{g}=n_{g}^{o b s}$, results in a steeper slope of $n_{Q S O}(z)$ at low redshifts $z \leq 2$ (fig. 3c). The curve labeled by 5 in fig. 3 is the number density of QSOs calculated without the threshold function $\left(\nu_{t h}=0\right)$. For comparison we have calculated also the $n_{Q S O}(z)$ without the threshold function for another minimal linear over density corresponding to a bound object at $z=0 \delta_{c}^{0}=1.33$ adopted by Nusser and Silk [14] (line labeled by 6 in fig. 3a).

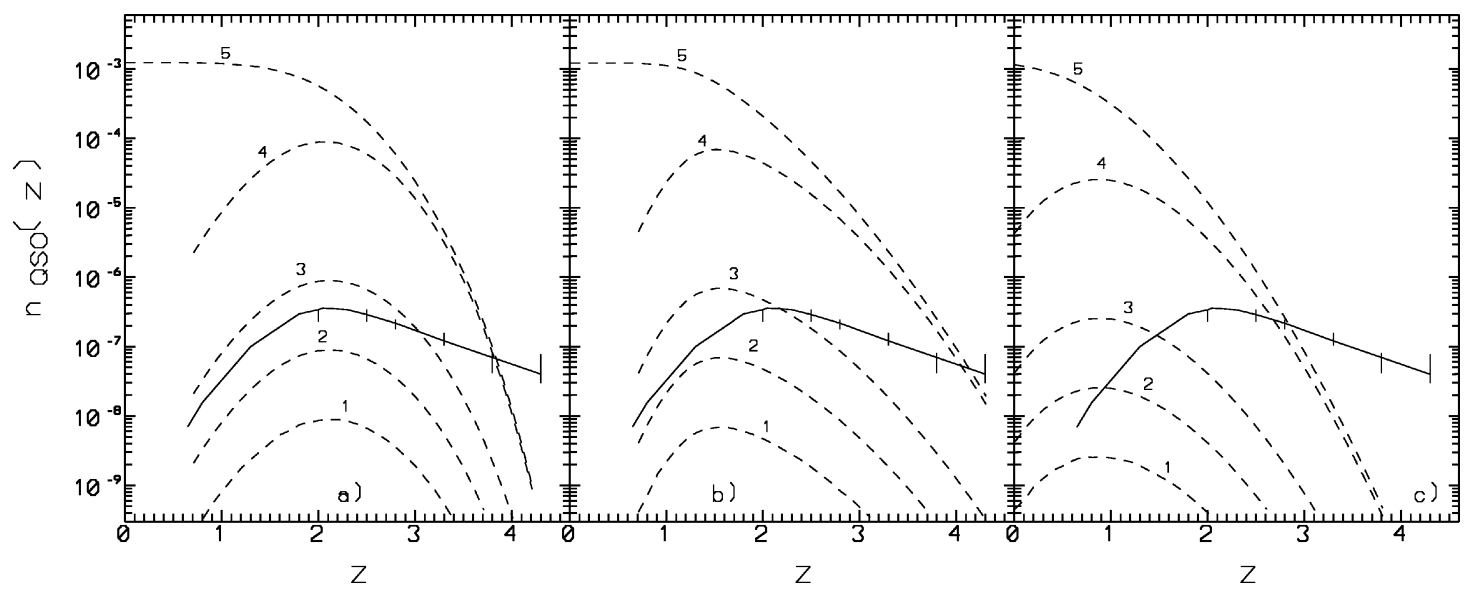

Fig. 2. Quasar abundance against redshift for CDM spectrum with $\Omega_{C D M}=0.9, \Omega_{b}=0.1, n=0.8$ (a), $n=0.7$ (b) and hybrid H+CDM model $\left(\Omega_{H D M}=0.3, \Omega_{C D M}=0.6, \Omega_{b}=0.1\right)$ for different values of the $\tau_{Q S O}=10^{4}, 10^{5}, 10^{6}, 10^{8}, 10^{10}$ years (dashed lines 1, 2, 3, 4, 5 respectively in each panel). Time delay $\Delta t$ is $5.2 \times 10^{8}$ years (a), 0 (b) and (c). The rest is the same as in fig. 1.

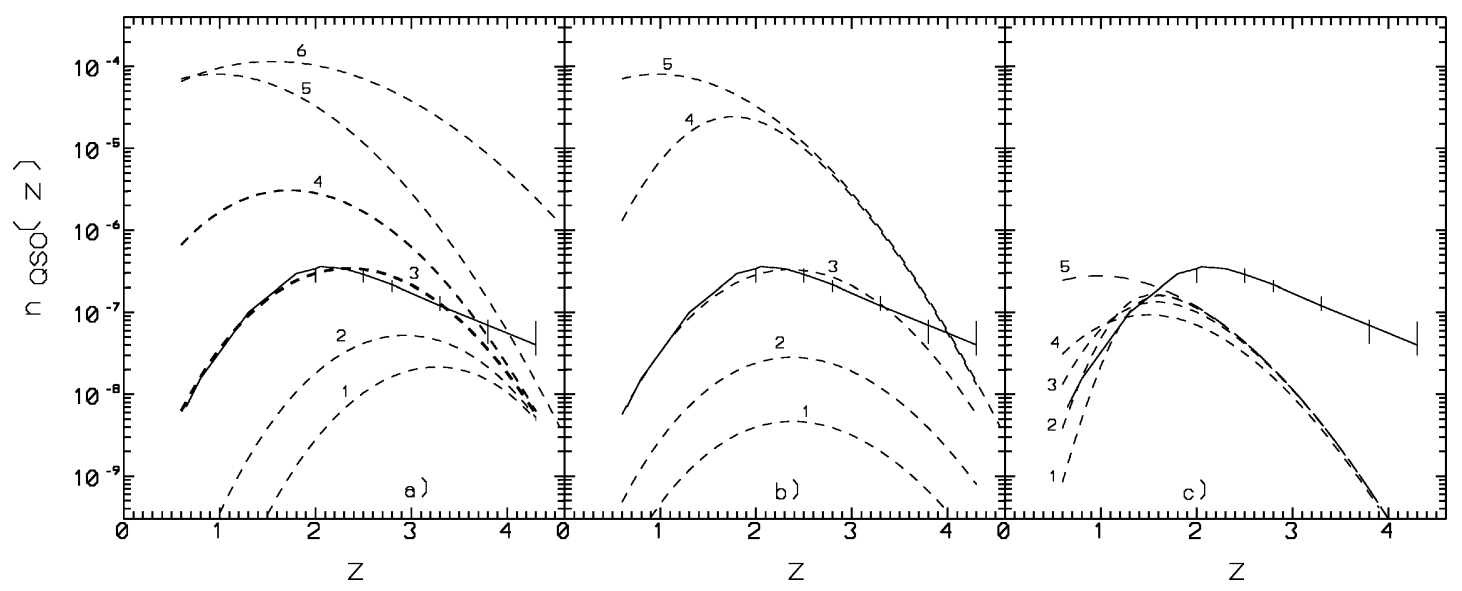

Fig. 3. Quasar abundance against redshift in a tilted CDM model $(n=0.7)$ for different parameters of the threshold function: (a) $-q=16$ (line 1), $q=12$ (2), $q=8$ (3), $q=4$ (4), without the threshold function (5), $\tau_{Q S O}=6.9 \times 10^{7}$ years, $\nu_{t h}=5.89 ;(\mathrm{b})-\nu_{t h}=10$ (line 1), $\nu_{t h}=8(2), \nu_{t h}=5.89(3), \nu_{t h}=3(4)$, without the threshold function $(5), \tau_{Q S O}=6.9 \times 10^{7}$ years, $q=8.08$; (c) - $q=16, \nu_{t h}=2.5$ (line 1), $q=12.4, \nu_{t h}=2.52(2), q=8, \nu_{t h}=2.6(3), q=4, \nu_{t h}=2.95$ (4), without the threshold function (5), $\tau_{Q S O}=2.4 \times 10^{5}$ years, ( $\nu_{t h}$ for the given $q$ was obtained from eq. 11). The rest is the same as in fig. 1 . 


\begin{tabular}{|c|c|c|c|c|}
\hline & \multicolumn{3}{|c|}{ CDM } & $H+C D M$ \\
\hline$n$ & 1 & 0.8 & 0.7 & 1 \\
\hline$\alpha \tau_{Q S O}$ (years) & $3.54 \times 10^{5}$ & $4.56 \times 10^{5}$ & $2.39 \times 10^{5}$ & $3.82 \times 10^{4}$ \\
$\Delta t$ (years) & $1.55 \times 10^{9}$ & $5.19 \times 10^{8}$ & 0.00 & 0.00 \\
$\nu_{t h}$ & 5.22 & 2.73 & 2.52 & 2.37 \\
$q$ & 1.95 & 6.60 & 12.4 & 4.30 \\
\hline
\end{tabular}

Table 2. Solutions $\alpha \tau_{Q S O}, \nu_{t h}$ and $q$ of a system of equations (10)-(11) for different spectra.

\begin{tabular}{|c|c|c|c|c|}
\hline & \multicolumn{3}{|c|}{ CDM } & $H+C D M$ \\
\hline$n$ & 1 & 0.8 & 0.7 & 1 \\
\hline$\alpha \tau_{Q S O}$ (years) & $5.73 \times 10^{4}$ & $1.83 \times 10^{7}$ & $6.91 \times 10^{7}$ & $1.64 \times 10^{8}$ \\
$\Delta t$ (years) & $1.3 \times 10^{9}$ & $5.19 \times 10^{8}$ & 0.00 & 0.00 \\
$\nu_{t h}$ & 1.09 & 7.26 & 5.89 & 4.95 \\
$q$ & 4.96 & 4.85 & 8.08 & 12.32 \\
$K$ & 7.2 & 0.02 & 0.006 & 0.003 \\
\hline
\end{tabular}

Table 3. Solutions $\alpha^{\prime} \tau_{Q S O}, \nu_{t h}, q$ of a system of equations (10) and the ratio $K$ of the number density of peaks which has passed through a quasar stage to one of bright galaxies.

\begin{tabular}{|c|c|c|c|c|c|}
\hline$\alpha \tau_{Q S O}$ (years) & $3.20 \times 10^{5}$ & $3.51 \times 10^{5}$ & $3.53 \times 10^{5}$ & $4.41 \times 10^{5}$ & $4.0 \times 10^{4}$ \\
$\Delta t$ (years) & 0.00 & 0.00 & 0.00 & 0.00 & 0.00 \\
$\nu_{t h}$ & 2.06 & 1.69 & 1.69 & 1.67 & 1.67 \\
$q$ & 8.71 & 9.28 & 9.28 & 9.31 & 9.31 \\
$\sigma_{0}$ & 2.38 & 2.77 & 2.78 & 2.81 & 2.82 \\
$\sigma_{1}$ & 1.48 & 0.22 & 0.17 & 0.02 & 0.002 \\
$\sigma_{2}$ & 2.37 & 0.43 & 0.34 & 0.03 & 0.004 \\
\hline
\end{tabular}

Table 4. Partial solutions $\alpha \tau_{Q S O}, \nu_{t h}, q, \sigma_{0}, \sigma_{1}$ and $\sigma_{2}$ of a system of equations (10)-(11).

Now, let us find for each model such values of $\alpha, \tau_{Q S O}$, $\nu_{t h}$ and $q$ which give a minimal divergence $n_{Q S O}(z)$ from the observable one and ensure the number density of massive galaxies at $z \approx 0$. In all cases analyzed here the amplitude of theoretical $n_{Q S O}(z)$ is roughly equal to the observable $n_{Q S O}^{o b s}(2.2) \simeq 3 \times 10^{-7} h^{3} M p c^{-3}$ when $\tau_{Q S O} \sim 10^{6}$ years that is $\ll t_{0}$. It means that $(8)$ is a good approximation of (10) in the range of $10^{-3} \leq \alpha \leq 1$ and that $n_{Q S O} \propto \alpha \tau_{Q S O}$. As a result, we can not find both values of $\alpha$ and $\tau_{Q S O}$ simultaneously but their product $\alpha \tau_{Q S O}$ only. Approximate solutions of a system of equations (10), (11) are obtained by using MathCad software package and presented in table 2 . The quasar abundances $n_{Q S O}(z)$ for them are shown in fig. 4 . As we can see no model explains the observable $n_{Q S O}^{o b s}(z)$ at high $z$. Therefore we have repeated the same procedure without the equation for the concentration of bright galaxies (11). The results are presented in table 3 and fig. 5 . In this case the parameter $\alpha$ has a different interpretation: it is the fraction of peaks which are selected by the threshold function of $p\left(\nu ; \nu_{t h}^{Q S O}, q^{Q S O}\right)$ and which pass through the quasar stage and are visible by the terres- tial observer. Let $\alpha^{\prime}$ denote it. The ratio of the number density of all peaks selected in such a way to the number density of bright galaxies

$$
K=\frac{\int_{0}^{\infty} p\left(\nu ; \nu_{t h}^{Q S O}, q^{Q S O}\right) N_{p k}(\nu) d \nu}{\int_{0}^{\infty} p\left(\nu ; \nu_{t h}^{g}, q^{g}\right) N_{p k}(\nu) d \nu}
$$

is calculated for each model and presented in table 3 . In all models, with the exception of the standard CDM, $K \ll 1$ and can be interpreted as $\alpha_{1}$ mentioned above, that is a fraction of the peaks-precursors of bright galaxies passed through the QSO stage. Thus, in these models the peaks resulting in bright galaxies through the QSO stage are higher at average than the main part of all peaks. The picture is inverse in standard CDM, where $K \approx 7$, and may mean that peaks of the galactic mass $M \geq 2 \times 10^{11} h^{-1} M_{\odot}$ survive after the QSO stage and become bright galaxies if their gravitational potential holes are sufficiently deep. Whether it is valid 
or not can be proved only by means of elaborate numerical simulations of the evolution of such peaks in different cosmological models. As follows from fig. 5, the theoretical number density of QSOs at low $z$ is satisfactory in all models. At high $z$ only the CDM model with $n=0.7$ gives the number density of QSOs sufficiently close to the observable one. But even this model does not explain the observable number density of QSOs at $z \geq 4$. Is it possible to explain the whole observable redshift distribution of quasar abundance given by Schmidt et al. [1] in such an approach, in principle?

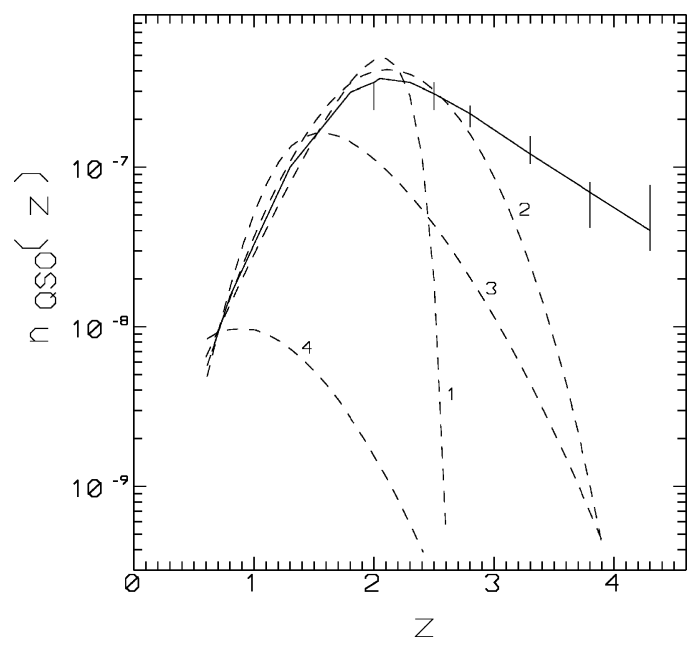

Fig. 4. Quasar abundance against redshift obtained by minimization of the divergence vector including also the number density of bright galaxies at $z \sim 0: 1-\mathrm{CDM}, n=1,2$ - CDM, $n=0.8,3-\mathrm{CDM}, n=0.7,4-\mathrm{H}+\mathrm{CDM}, n=1$.

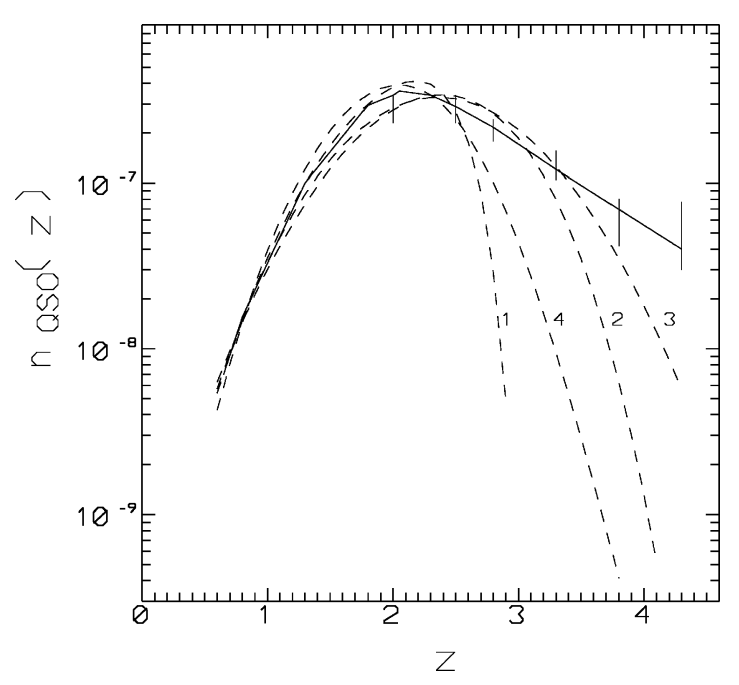

Fig. 5. Quasar abundance against redshift obtained by minimization of the divergence vector without the abundance of bright galaxies: $1-\mathrm{CDM}, n=1,2-\mathrm{CDM}, n=0.8,3$ - CDM, $n=0.7,4-\mathrm{H}+\mathrm{CDM}, n=1$.

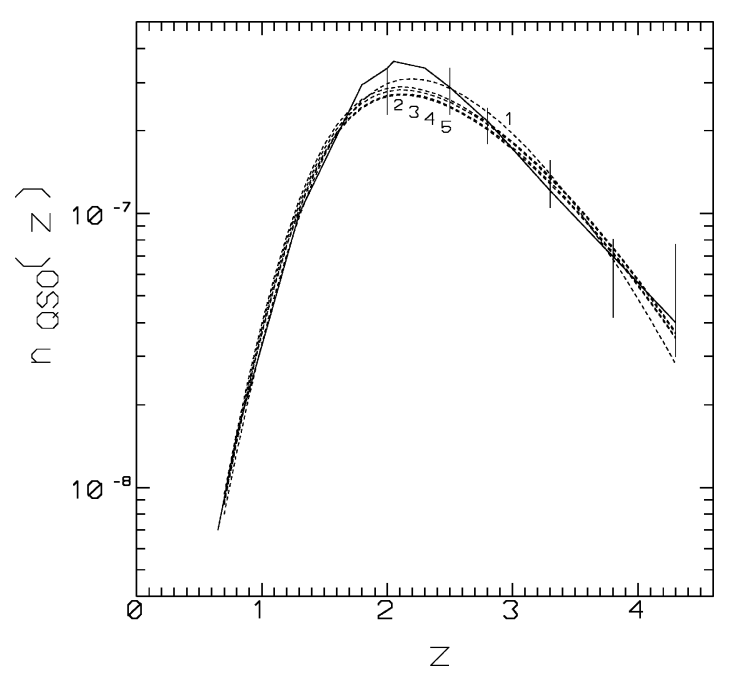

Fig. 6. Quasar abundance against redshift for the found momenta of spectra, parameters of threshold function and $\alpha \tau_{Q S O}$.

\section{B. Finding the phenomenological spectrum}

To answer this question we attempted to solve a system of equations (10), (11) with respect to the unknown values $\sigma_{0}, \sigma_{1}, \sigma_{2}, \alpha \tau_{Q S O}, \nu_{t h}$ and $q$. The time delay $\Delta t$ is assumed to be equal to zero. Solutions are obtained in the same way and are presented in table 4. Quasar abundances $n_{Q S O}(z)$ for them are shown in fig. 6. and match the observable data quite well. As we can see, the main feature of all solutions is the relation between momenta: $\sigma_{1}<\sigma_{2}<\sigma_{0}$. What kind of spectrum have such relations between their momenta on the galaxy scale? We attempted to modify the standard CDM spectrum on small scale by force of reducing and enhancing its power in order to obtain momenta of spectra from table 4 . Locations and amplitudes of such modifications were unknown parameters and were found from 3 equations for given momenta $\sigma_{1}, \sigma_{2}, \sigma_{0}$ using MathCad software package. It was successful for momenta from column 1 in table 4 only, for which parameter $\gamma \equiv \sigma_{1}^{2} / \sigma_{0} \sigma_{2}=0.3$. The phenomenological power spectrum of density fluctuations obtained in such a way is

$$
P_{p h}(k)=A k T_{C D M}^{2}\left\{\begin{array}{cl}
1, & k<k_{0}, \\
e^{1-\left(k / k_{0}\right)^{p}}, & k_{0} \leq k \leq k_{1} \\
e^{1-\left(k_{1} / k_{0}\right)^{p}}, & k>k_{1},
\end{array}\right.
$$

where $k_{0}=0.48 h M p c^{-1}, k_{1}=0.82 h M p c^{-1}, p=2.3, A$ is the same as in standard CDM. The spectrum has shelflike reducing of power at $k \sim 0.7 h \mathrm{Mpc}^{-1}$ in comparison with standard CDM normalised to the COBE $10^{\circ}$ angular scale temperature anisotropy of CMB $\left(P_{1 p h}(k)\right.$ spectrum in fig. 7). The correlation function of galaxies for it does not contradict observable data. Other cosmological consequences of such a spectrum are being still investigated. 


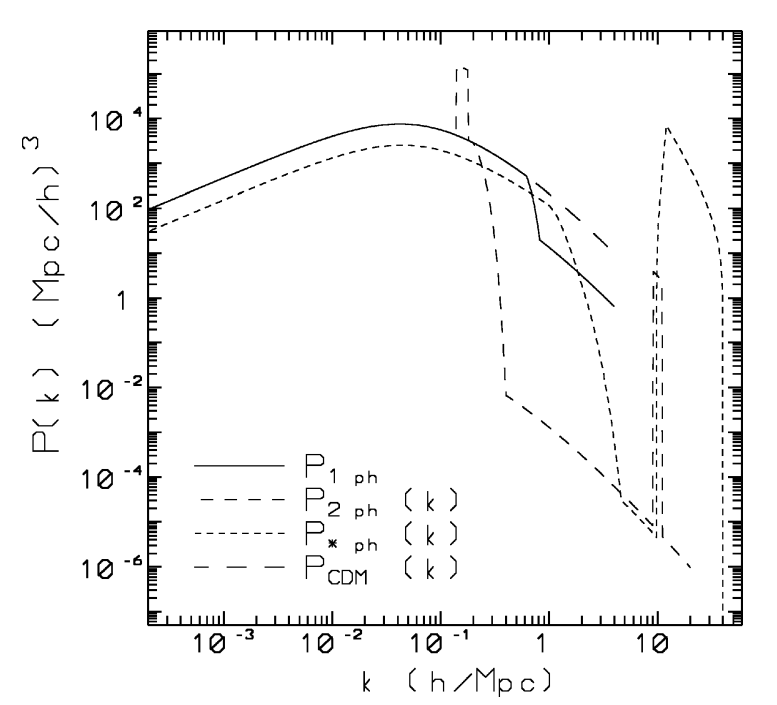

Fig. 7. The power spectrum of density fluctuation normalised to produce COBE $\Delta T / T$ at $10^{\circ}$ angular scale for standard CDM, its modification for momenta from column 1 in table $4\left(P_{1 p h}\right)$, the same for momenta from column 2 in table $4\left(P_{2} p h\right)$ and the phenomenological power spectrum with $\sigma_{0}=2.37, \sigma_{1}=1.16, \sigma_{2}=1.89\left(\alpha \tau_{Q S O}=4.4 \times 10^{5}\right.$ years, $b=1.72$ ) obtained by the modification of standard CDM normalised to $\sigma_{8}=b_{g}^{-1}[34,35]\left(P_{* p h}\right)$.

The parameter $\gamma$ for momenta from columns $2-5$ in table 4 is extremely small $(0.04-0.0004)$, so that the phenomenological power spectrum for them, obviously, must be extraordinary too. Indeed, the spectrum with momenta from column $2\left(P_{2} p h(k)\right.$ spectrum in fig. 7$)$ has two narrow bumps at $k \approx 0.02$ and $\approx 10 h \mathrm{Mpc}^{-1}$ and shelf-like reducing of power at $k \sim 0.03 h \mathrm{Mpc}^{-1}$ in comparison with standard CDM. Since its correlation function of bright galaxies strongly contradicts the observable one, the solutions from columns $2-5$ in table 4 can be interpreted as meaningless from the physical point of view. So, only the spectra with momenta from column $1(\gamma \approx 0.3)$, or close to them, are cosmologically significant.

\section{Estimation of fraction of host galaxies}

Masses of galaxies which pass through the quasar stage (host galaxies) are $M_{g} \geq 2 \times 10^{11} h^{-1} M_{\odot}$, so that it is coordinated with the black hole accretion models of the quasar phenomenon $[19,20,32,33]$. An optical luminosity of the bright quasars is $L \geq 10^{47} h^{-2} \mathrm{erg} / \mathrm{s}$. Their lifetime multiplied by $\alpha$ in all cases is $\alpha \tau_{Q S O} \geq 10^{5}$ years, because we denote $\tau_{Q S O}=10^{5} \tau_{5} / \alpha$, where $\tau_{5}$ is $\alpha \tau_{Q S O}$ in the units of $10^{5}$. If the fraction of the black hole mass converted into optical radiation is $\epsilon$, the ratio of the quasar's mass to that of the host galaxy is $F$, then

$$
M_{g} \approx 2 \times 10^{5} L_{47} \tau_{5} /(\alpha \epsilon F) h^{-2}
$$

which gives

$$
\alpha \approx 10^{-3} L_{47} \tau_{5} /\left(\epsilon_{0.1} F_{0.01}\right) h^{-1}
$$

where $\epsilon_{0.1} \equiv \epsilon / 0.1, F_{0.01} \equiv F / 0.01, L_{47} \equiv L / 10^{47}$.

Thus, in the models with phenomenological power spectrum and spectra tested here only less than $1 \%$ of massive galaxies pass through quasar stage and are visible by the terrestial observer.

\section{DISCUSSION}

Recently we $[34,35]$ carried out similar calculations of the evolution of the quasar number density for the same spectra (CDM with $\mathrm{n}=1,0.8,0.7$ and $\mathrm{H}+\mathrm{CDM}$ ) normalised to $\sigma_{8}=b_{g}^{-1}$, where galactic biasing parameter $b_{g}$ was calculated in the framework of Gaussian statistics of density peaks $[15,36]$ and was equal to $1.40,1.47,1.53$ and 1.76 respectively. Comparing the results of this work with the previous ones it follows that the dependence of the quasar number density on $z$ is very sensitive to the normalisation of the spectrum in all models. In our previous papers the standard CDM spectrum modified on small scale for reproducing the observable data on the number density of QSOs at different redshifts has reduced the power on the scale $1 \leq k \leq 10 h \mathrm{Mpc}^{-1}$ and bumped on the scale $k \sim 10 h M p c^{-1}$ (dotted line in fig. 7). Its difference from the spectrum shown in fig. 7 is caused by a different power of the initial CDM spectrum on $k \leq 1 h M p c^{-1}$ as a result of a different normalisation.

It should be remarked here that though formally this method is sensitive to spectrum on a small scale up to $k \sim 10 h M p c^{-1}$ when it is smoothed by the Gaussian filter function with $R_{f}=0.35 h^{-1} M p c$ (or top-hat $\left.R_{T H}=0.6 h^{-1} M p c\right)$ nevertheless we are not sure that calculation of peak number density of galaxy scale is exact for cases of spectrum like standard CDM and, especially, CDM + short wave bump models. This is concerned mainly with the well known problem 'cloud-incloud' which is unresolved as yet in the frame of peak formalism used here.

Now, let us compare our results with those by Nusser and Silk [14]. The curve $n_{Q S O}(z)$ for $\delta_{c}^{o}=1.33$ (line 6 in fig. 3) without the threshold function, recalculated to $\alpha \tau_{Q S O}=10^{8}$ years, coordinates with the corresponding curve in fig. 2 in the work by Nusser \& Silk [14] quite satisfactorily at high z. A certain divergence of these curves at low $\mathrm{z}$ are caused mainly by varying the definitions of the mass of peaks.

In our work the value $\delta_{c}^{o}=1.68$ corresponding to the collapse of spherical-symmetrical peaks is adopted. Therefore, in our approach the QSOs are formed in spheroidal peaks only. The fraction of spheroidal peaks is $\leq 4 \%$ [18], the fraction of massive galaxies with $M \geq 2 \times 10^{11} h^{-1} M_{\odot}$ passed through the quasar stage in all cases does not exceed $1 \%$. So, the assumption that QSOs appear only in the spherical peaks of density fluctuations is not contradictory in our approach. But its conformity to the reality should be proved or objected 
by elaborated numerical simulations of the evolution of QSOs which start from the initial conditions determined at the linear stage of the evolution of peaks. Substantiations of the value of $\delta_{c}^{o}$ as well as of the dependence or independence of both the quasar lifetime $\tau_{Q S O}$ and the time delay $\Delta t$ on the redshift $z$ will be the subject of future works.

We put constraints on the cosmological models based on their ability to reproduce the rise of the quasar abundance up to redshifts $\approx 2.2$ and following a fall up to $\approx 4.7$ assuming that the quasar nature is the same at different redshifts and $\tau_{Q S O}, \Delta t$ and $\alpha$ do not depend on z. Moreover, if we have only 3 unknown values $\alpha \tau_{Q S O}$, $\nu_{t h}$ and $q$ in equation system (10-11) then we can not reproduce the $n_{Q S O}^{o b s}(z)$ given by Boyle et al. [17] and Schmidt et al. [1] with sufficient accuracy in the models tested here. But when $\sigma_{0}, \sigma_{1}$ and $\sigma_{3}$ are supposed to be the unknown values too, such a reproduction is possible. Apparently, for other models of quasars, which explain the rise and fall of the $n_{Q S O}^{o b s}(z)$ by astrophysics of quasars (e.g. [37]), the constraints on the cosmological models may be different. But it is the subject of separate work. Here we only confirm that such a dependence of the quasar abundance on $\mathrm{z}$ can be explained in the framework of the simplest astrophysical models of quasars, which look rather plausible and motivated in order to be laid as the basis of a similar research.

The phenomenological power spectrum obtained here (III.B) has a shelf-like reducing of power at $k \sim$ $0.7 h M p c^{-1}$. Such spectra with a broken scale invariance is generated in some inflationary scenarios (e.g. [38-41]). The typical scale and the height of the shelf-like reduction determined here can be connected with some constants which characterise the underlying inflationary models.

If we treat the used observational data more critically and suppose that $n_{Q S O}^{o b s}(z)$ given by Schmidt et al. [1] and Boyle et al. [17] is just the lower limit for the number density of QSOs at all $z$ then the next constraints follow from the presented results: on the small scale the initial power spectrum of density fluctuations is nearly CDM with $n \geq 0.7, b_{g} \leq 2.3$ and $\alpha \tau_{Q S O} \geq 5 \times 10^{5}$ years, $\Delta t \leq 5 \times 10^{8}$ years.

\section{CONCLUSIONS}

Thus, the main assumption that a quasar phenomenon is an active short term stage of the evolution of some small fraction of massive galaxies with $M \geq 2 \times$ $10^{11} h^{-1} M_{\odot}$ and appears in peaks of random Gaussian density fluctuation field allows to explain the general feature of $n_{Q S O}^{o b s}(z)$ given by Boyle et al. [17] and Schmidt et al. [1]: rapidly increasing the number density from $z \approx 0$ to $z \sim 2.2$ up to the value of $\sim 3 \times 10^{-7} h^{3} M p c^{-3}$ and following monotonous decreasing on $z>2.5$. Apparently, a constant quasar abundance from $z \approx 2$ to 4 [42] can not be explained under these assumptions.

If the nature of bright QSOs is the same at different redshifts $\left(\tau_{Q S O}, \Delta t, \alpha\right.$ are constants) then any model spectrum tested here such as a standard CDM spectrum $\left(\Omega_{C D M}=0.9, \Omega_{b}=0.1\right)$, the tilted one with $n=0.7,0.8$ and the hybrid $\mathrm{H}+\mathrm{CDM}\left(\Omega_{C D M}=0.6, \Omega_{H D M}=0.3\right.$, $\Omega_{b}=0.1, n=1$ ) explains the number density of such QSOs in the redshift range of $z \approx 0.5-2.2$, but in the range of $z \approx 0.5-3.5$ only the tilted CDM with $n=0.7$ $\left(\tau_{Q S O} \approx 7 \times 10^{7}\right.$ years, $\left.\alpha_{1} \approx 0.01, \Delta t=0\right)$ does explain it, and in the range of $z \approx 0.5-4.7$ - none of them do. Consequently, more crucial for testing of spectra is the number density of QSOs on high $z$.

The exact reproduction of the number density of QSOS at $z=0.5-4.7$ under the above mentioned assumptions can be done in the flat cosmological model with the density fluctuation power spectrum which gives $\sigma_{0} \approx 2.4-$ 2.8 and $\gamma \leq 0.3$ at the galaxy scale $\left(R_{f}=0.35 h^{-1} \mathrm{Mpc}\right)$. It predicts the QSO's lifetime $\tau_{Q S O} \approx(3.5-4.5) \times 10^{5} / \alpha$ years, where $\alpha$ is a fraction of massive galaxies passing through the quasar stage. If physically motivated quasar lifetime is $\sim 10^{7}-10^{8}$ years, then $\alpha \approx 0.01-0.001$ which matches the estimations of $\alpha$ done above.

Thus, redshift distribution of quasar abundance is very sensitive to the amplitude and slope of the initial power spectrum of density perturbations on the galaxy scale. But it can be an effective test when complete number density of QSOs with the mass larger than the fixed value will be known with confidence for all redshifts and physical parameters connected with the nature of the quasar phenomenon (the quasar lifetime $\tau_{Q S O}$, time delay $\Delta t$, fraction of galaxies which pass through the quasar stage $\alpha$, linear amplitude of peaks $\delta_{c}^{o}$ collapsing just now and the threshold function) will be substantiated. The latter calls for a detailed investigation of the connection of the quasar phenomenon with the initial configuration of density fluctuation by means of numerical simulations of its evolution.

We acknowledge useful discussions with B. Hnatyk, V. N. Lukash and B. V. Komberg.
[1] M. Schmidt, D. P. Schneider, J. E. Gunn, in The Space Density of Quasars, ed. D. Crampton (SF: ASP) (1991, p. 109).

[2] M. J. Davis, F. J. Summers, D. Schlegel, Nature 359, 393 (1992).

[3] A. N. Taylor, M. Rowan-Robinson, Nature 359, 396
(1992).

[4] A. van Dalen, R. K. Schaefer, ApJ 398, 33 (1992).

[5] R. Cen, N. Yu. Gnedin, L. A. Kofman, J. P. Ostriker, ApJ 399, L11 (1992).

[6] D. Yu. Pogosyan, A. A. Starobinsky, MNRAS 265, 507 (1993). 
[7] L. A. Kofman, N. Y. Gnedin, N. A. Bahcall, ApJ 413, 1 (1993).

[8] Б. С. Новосядлий, Кінематика і фізика небесних тіл 10, 13 (1994).

[9] B. I. Hnatyk, V. N. Lukash, B. S. Novosyadlyj, A\&A, 300, 1 (1995).

[10] W. H. Press, P. Schechter, ApJ 187, 425 (1974).

[11] A. Blanchard, T. Buchert, R. Klaffl, A\&A 267, 1 (1993).

[12] G. F. Smoot, C. L. Bennett, A. Kogut, E. L. Wright et al., ApJ 396, L1 (1992).

[13] A. Kashlinsky, ApJ 406, L1 (1993).

[14] A. Nusser, J. Silk, ApJ 411, L1 (1993).

[15] J. M. Bardeen, J. R. Bond, N. Kaiser, A. S. Szalay, ApJ, 304, 15 (1986).

[16] C. Ma, E. Bertschinger, ApJ 434, L5 (1994).

[17] B. J. Boyle, T. Shanks, B. A. Peterson, MNRAS 235, 935 (1988).

[18] A. G. Doroshkevich, Astrophysics 6, 581 (1970).

[19] E. L. Turner, AJ 101, 5 (1991).

[20] A. Loeb, F. A. Rasio, ApJ 432, 52 (1994).

[21] M. J. Davis, J. Huchra, ApJ 254, 437 (1982).

[22] MathCAD. Version 2.5. User Guide (MathSoft Ins., Cambridge, 1989).

[23] J. A. Holtzman, ApJS 71, 1 (1989).

[24] C. L. Bennett, A. Kogut, G. Hinshaw, A. I. Banday, E. l. Wright et al., ApJ 436, 423 (1994).

[25] E. L. Wright, G. F. Smoot, C. L. Bennett, P. M. Lubin, ApJ 436, 443 (1994).

[26] M. L. Wilson, J. Silk, ApJ 243, 14 (1981).

[27] E. Martinez-Gonzales, J. Sanz, ApJ 347, 11 (1989).
[28] B. S. Novosyadlyj, Astron. and Astroph. Transactions 10, 85 (1996).

[29] M. White, D. Scott, J. Silk, Annu. Rev. Astron. Astrophys. 47, 426 (1994).

[30] F. C. Adams, I. R. Bond, K. Freese, I. A. Frieman, A. V. Olinto, Phys. Rev. D 47, 426 (1993).

[31] E. F. Bunn, D. Scott, M. White, ApJ 441, L9 (1995).

[32] G. Efstathiou, M. J. Rees, MNRAS 230, 5 (1988).

[33] A. Kashlinsky, B. J. T. Jones, Nature 349, L1 (1991).

[34] Yu. B. Chornij, B. S. Novosyadlyj, Astron. and Astroph. Transactions 10, 77 (1996).

[35] Б. С. Новосядлий, Ю. Б. Чорній, Кінематика і фізика небесних тіл 12, 30 (1996).

[36] Б. І. Гнатик, В. Н. Лукаш, Б. С. Новосядлий, Кінематика і фізика небесних тіл 7, 48 (1991).

[37] M. Haehnelt, M. J. Rees, MNRAS 263, 168 (1993).

[38] A. Amendola, F. Occhionero, D. Saez, ApJ 349, 399 (1990).

[39] S. Gottlöber, V. Müller, A. A. Starobinsky, Phys. Rev. D 43, 2510 (1991).

[40] A. A. Starobinsky, Pis'ma Zh. Eksp. Teor. Fiz. 55, 477 (1992).

[41] P. Peter, D. Polarski, A. A. Starobinsky, Phys. Rev. D 50, 4827 (1994).

[42] M. Irwin, R. G. McMahon, C. Hazard, in The Space Density of Quasars, ed. D. Crampton (SF: ASP), (1991, p. 117).

[43] The parameter $t_{1}$ of transfer function fitting the formula by Holtzman [23] is accepted equal to 1 .

\title{
КОНЦЕНТРАЩЯ КВАЗАРІВ ЯК ТЕСТ ПОЧАТКОВОГО СПЕКТРУ ПОТУЖНОСТІ НА МАЛИХ МАСШТАБАХ
}

\author{
Б. Новосядлий, Ю. Чорній \\ Астрономічна обсерваторія Лъвівсъкого державного університету імені Івана Франка \\ Україна, UА-290005, Лъвів, вул. Кирила і Мефоділ, 8
}

\begin{abstract}
Вивчається залежність концентрації яскравих квазарів на різних червоних зміщеннях $\left(n_{Q S O}(z)\right)$ від початкового спектру потужності. Приймається, що квазарний феномен є ранньою короткочасною стадією еволюції масивних галактик з масою $M \geq 2 \times 10^{11} h^{-1} M_{\odot}$. Тривалість цієї квазарної стадії, котру проходить деяка частина галактик $\alpha, \mathrm{\epsilon}$ визначена методом мінімізації відхилення теоретичної концентрації квазарів на різних червоних зміщеннях для заданих початкових спектрів від спостережуваної [1]. Показано, що найближчою до спостережуваної є концентрація квазарів на $0.7 \leq z \leq 3.5$, отримана для нахиленої CDM моделі $\left(\Omega_{b}=0.1, n=0.7\right)$. Квазарна стадія триває $\sim 7 \times 10^{7} / \alpha$ років і починається відразу після появи перших протипотоків у беззіткнювальній компоненті і ударної хвилі в газі.

Також вивчалась можливість реконструкції початкового спектру потужності на малих масштабах на основі спостережуваних даних по концентрації квазарів на різних $z$. Такий реконструйований спектр у порівнянні із стандартним CDM спектром має сходинкоподібне зменшення потужності на $k \geq 0.5 h M p c^{-1}$.
\end{abstract}

